\title{
A FRAMEWORK FOR GENERATING REALISTIC SYNTHETIC SEQUENCES OF TOTAL INTERNAL REFLECTION FLUORESCENCE MICROSCOPY IMAGES
}

\author{
Seyed Hamid Rezatofighi ${ }^{1,2}$, William T. E. Pitkeathly ${ }^{3}$, Stephen Gould ${ }^{1}$, Richard Hartley ${ }^{1,4}$, \\ Katarina Mele ${ }^{2}$, William E. Hughes ${ }^{5,6}$ and James G. Burchfield ${ }^{5}$ \\ ${ }^{1}$ College of Engineering and Computer Science, The Australian National University, Australia, \\ ${ }^{2}$ Quantitative Imaging Group, CSIRO Mathematics, Informatics and Statistics, Australia, \\ ${ }^{3}$ Physical Sciences of Imaging in the Biomedical Sciences, Doctoral Training Centre, University of Birmingham, UK, \\ ${ }^{4}$ National ICT (NICTA), Australia, \\ ${ }^{5}$ The Garvan Institute of Medical Research, Australia, \\ ${ }^{6}$ Department of Medicine, St. Vincents Hospital, Australia.
}

\begin{abstract}
Since generation of reliable ground truth annotation of fluorescence microscopy sequences is usually a laborious and expensive task, many proposed detection and tracking methods have been evaluated using synthetic data with known ground truth. However, differences between real and synthetic images may lead to inaccurate judgment about the performance of an algorithm. In this paper, we present a framework for generating realistic synthetic sequences of total internal reflection fluorescence microscope (TIRFM) through simulation of the image formation process and accurate measurement and dynamic models. The sequences generated using this framework appropriately reflect the complexities existing in real TIRFM sequences.
\end{abstract}

Index Terms - TIRFM, Synthetic data, Background estimation, Vesicle shape deformation, Dynamic model.

\section{INTRODUCTION}

Total internal reflection fluorescence microscopy (TIRFM) is an imaging technique that enables the selective excitation of fluorophores within a few hundred nano-meters of the plasma membrane of a cell. Therefore it is ideal for visualizing subcellular structures such as vesicles that are on or close to the plasma membrane [1]. Manual analysis of such data sets is a laborious task which is subject to judgement bias. Hence, the development of automated methodologies which are able to detect and track subcellular structures would provide a crucial framework for understanding many biological process.

Recently, many detection and tracking techniques have been proposed in different fluorescence microscopy applications $[2,3,4,5,6]$. However, without the ground truth, the performance of these method can not be correctly evaluated on real sequences. But ground truth is difficult to obtain due to lack of accuracy and speed in manual procedure. To this end, the performance of these algorithms is quantitatively evaluated using synthetic data where the ground truth is known.
In most previous works [2, 3, 4, 5], some simple assumptions are used in generating synthetic data such as sequences with constant signal-to-noise ratio (SNR) and without background structures, particles with fixed shapes and with Gaussian spatial intensity modeling point spread (PSF) function of imaging system. Although these assumptions may ease the procedure of generation of synthetic data, they do not reflect the complexities existing in real sequences. For example, it has been shown that many detection algorithms fail in presence of background structures (clutter) [2]. Furthermore, since the algorithms' parameters can be tuned for a specific SNR, a synthetic data with constant SNR can not properly challenge the algorithms whilst real data contains sequences with spatiotemporally varying SNR.

In this paper, we propose a general framework for generating synthetic TIRFM sequences based on realistic measurements and dynamics models. The sequences generated using this framework appropriately reflect the difficulties existing in real TIRFM sequences*. Therefore, the sequences can be an apt evaluator for detection and tracking methods.

\section{METHODS}

\subsection{Measurement Model}

TIRFM uses an evanescent field to exclusively illuminate the sample within a few hundred nanometres from the imaging surface [7]. In cell biology studies the evanescent field is produced at the surface of a glass cover-slip on which cells reside, in some aqueous medium. The field behaves as a sheet of light whose intensity decays exponentially with distance from the imaging surface defined by $I_{z}=I_{0} \exp (-z / \zeta)$, where $I_{z}$ is the intensity of the field at distance $z, I_{0}$ is the intensity of the field at its origin, $z_{0}$, and $\zeta$ is the decay constant.

Real TIRFM sequences generally contain hundreds of dynamic subcellular structures which appear as bright spot

${ }^{*}$ The code and the generated sequences are available upon request by email to: hamid.rezatofighi@anu.edu.au,or at the first author's website. 
like objects, $\mathcal{O}$, superimposed onto a spatially and temporally varying background $\mathcal{B}$. Noting this fact that the background is additive to the sequences [2,3], the noise-free intensity of the sequences $\mathfrak{I}$ observed at pixel $\mathrm{X}=(x, y)^{T}$ and at time $t$ can be modeled using the following equation.

$$
\mathfrak{I}(\mathrm{X}, t)=\mathcal{B}(\mathrm{x}, t)+\mathcal{O}(\mathrm{x}, t),
$$

For TIRFM, the object intensity model observed at pixel $\mathrm{X}$ and at time $t$ can be mathematically defined as

$$
\mathcal{O}(\mathrm{x}, t)=\sum_{k=1}^{n_{t}} I_{O}\left(z_{t}^{k}\right)\left(\left.O\left(\mathrm{x} ; \mathrm{x}_{t}^{k}, \Sigma_{t}^{k}\right) * P_{s}\right|_{z=z_{t}^{k}}\right)
$$

where $n_{t}$ is number of objects at frame $t, *$ denotes the convolution of the region occupied by an object, $O$, with the threedimensional point spread function (PSF), $P_{s}$, of the objective lens at the object's depth $z_{t}^{k} \cdot \mathrm{X}_{t}^{k}=\left(x_{t}^{k}, y_{t}^{k}\right)^{T}$ is the position of each object $k$ at time $t$ and $\Sigma_{t}^{k}$ is a shape matrix which can be different for each object and evolves over the time with the state of the object. $I_{O}(z)$ is the intensity of fluorescence of the object obtained by the decaying exponential defined above.

In real sequences, it can be observed that subcellular structures become elongated in the same direction of their motions due to various biophysical factors. This phenomenon is observable in dynamic vesicles as they are seen as elongated 'comet' or 'tear drop' shaped objects when they move (Fig. 3) [8,9]. Therefore, to improve the particle model, an evolving procedure for the shape matrix based on the state of the object is proposed. In order to generate different shapes such as circle, ellipse and tear drop using a model, the shapes of objects are constructed in a piecewise manner using two half ellipses with different shape matrices which can be evolved based on their dynamics (Fig. 1).

Suppose $\mathcal{E}(\mathrm{X} ; \mathrm{M}, \Sigma)=\left\{\mathrm{X} \mid(\mathrm{X}-\mathrm{M})^{T} \Sigma^{-1}(\mathrm{X}-\mathrm{M}) \leqslant 1\right\}$ and $\mathcal{S}(\mathrm{X} ; \mathrm{M}, \mathrm{C})=\left\{\mathrm{x} \mid \mathrm{C}^{T}(\mathrm{x}-\mathrm{M}) \geqslant 0\right\}$ represent an ellipsoids volume (elliptical surface in $2 \mathrm{D}$ case), and a half space, respectively. Then $O$ can be obtained as follows.

$$
\begin{aligned}
O\left(\mathrm{x} ; \mathrm{x}_{t}^{k}, \Sigma_{t}^{k}\right)= & \mathcal{E}\left(\mathrm{x} ; \mathrm{x}_{t}^{k}, \Sigma_{t, 1}^{k}\right) \cap \mathcal{S}\left(\mathrm{x} ; \mathrm{x}_{t}^{k}, \mathrm{C}_{\theta}\right) \\
& \cup \mathcal{E}\left(\mathrm{x} ; \mathrm{x}_{t}^{k}, \Sigma_{t, 2}^{k}\right) \cap \mathcal{S}\left(\mathrm{x} ; \mathrm{x}_{t}^{k},-\mathrm{C}_{\theta}\right)
\end{aligned}
$$

where

and

$$
\mathrm{C}_{\theta}=\left[\begin{array}{ll}
\cos \theta_{t}^{k} & \sin \theta_{t}^{k}
\end{array}\right]^{T}, \quad \tan \theta_{t}^{k}=\frac{\dot{y}_{t}^{k}}{\dot{x}_{t}^{k}},
$$

$$
\Sigma_{t, l}^{k}=\left(\Theta_{t}^{k}\right)^{T} \Gamma_{t, l}^{k} \Theta_{t}^{k}, \quad l=1,2 .
$$

Parameters $\Theta_{t}^{k}$ and $\Gamma_{t}^{k}$ are the rotation and axes matrices respectively and calculated as

$$
\begin{aligned}
\Theta_{t}^{k} & =\left[\begin{array}{cc}
\cos \theta_{t}^{k} & \sin \theta_{t}^{k} \\
-\sin \theta_{t}^{k} & \cos \theta_{t}^{k}
\end{array}\right], \\
\Gamma_{t, 1}^{k} & =\left[\begin{array}{cc}
\left(\sigma_{x}^{k}\right)^{2} & 0 \\
0 & \left(\sigma_{y}^{k}\right)^{2} \bar{f}\left(\left\|\dot{\mathrm{x}}_{t}^{k}\right\|\right)
\end{array}\right], \\
\Gamma_{t, 2}^{k} & =\left[\begin{array}{cc}
\left(\sigma_{x}^{k}\right)^{2} f\left(\left\|\dot{\mathrm{x}}_{t}^{k}\right\|\right) & 0 \\
0 & \left(\sigma_{y}^{k}\right)^{2} \bar{f}\left(\left\|\dot{\mathrm{x}}_{t}^{k}\right\|\right)
\end{array}\right] .
\end{aligned}
$$

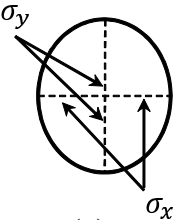

(a)

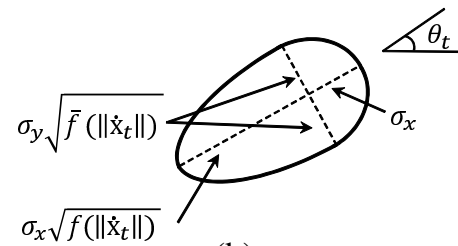

(b)
Fig. 1. The proposed evolving shape procedure for a object (a) before any motion and (b) after movement in direction $\theta_{t}$.

The $\sigma_{x}^{k}$ and $\sigma_{y}^{k}$ determine initial size of each object for each axis prior to any motion. The $\dot{\mathrm{x}}_{t}^{k}=\left(\dot{x}_{t}^{k}, \dot{y}_{t}^{k}\right)^{T}$ is the velocity element of each object at frame $t$. The $f($.$) and \bar{f}($.$) are$ deformation functions which distort the shape based on the magnitude of velocity $v=\|\dot{\mathrm{X}}\|$. To keep the rate of change of axes constant, the $\bar{f}\left(\right.$.) should be equal to $f^{-1}($.). Moreover, the function must fulfill the $f(0)=\bar{f}(0)=1$. The form of this function is different for each biological application and depends on many biophysical factors such as viscosity, elasticity and osmotic pressure of the membrane of the structures $[8,9]$. In this paper, we simplify this complexity and approximate it by a quadratic exponential function as the follows.

$$
f\left(\left\|\dot{\mathrm{x}}_{t}^{k}\right\|\right)=\exp \left(\frac{\left(\dot{x}_{t}^{k}\right)^{2}+\left(\dot{y}_{t}^{k}\right)^{2}}{\gamma^{2}}\right)
$$

The parameter $\gamma$ summarizes all biophysical factors in a value and controls the effect of the velocity on this deformation.

In this paper, the point spread function is modeled using an analytical expression for the diffraction pattern of light through a pupil with a perfect aberration free lens [10].

$$
\begin{aligned}
& P_{s}(u, v)=\left|2 \int_{0}^{1} P(\rho) J_{0}(\rho v) \exp \left(i u \rho^{2} / 2\right) \rho d \rho\right|^{2} \\
& u=2 \pi N A^{2} z / \lambda, \quad v=2 \pi N A r / \lambda
\end{aligned}
$$

where $P(\rho)$ is a pupil function with an aperture radius $R, J_{0}($.$) is a first order Bessel function, \rho=r / R, r=$ $\sqrt{x^{2}+y^{2}}, N A$ is the numerical aperture of the lens, $\lambda$ is wavelength of the light diffracted by aperture.

This function simulates the slight defocus effects of particles at different distances from the focal plane which are seen when $\zeta$ is large $(\sim 200-300 \mathrm{~nm})$. These are observable in real TIRFM for bright particles relatively distant from the evanescent field origin.

To extract the background of real TIRFM sequences, we use the maximum possible h-dome (MPHD) method [11]. This method extracts all regional peaked domes from each frame and enhances them to their maximum possible height. Since the objects of interest appear as regional domes (the second term, $\mathcal{O}$, in Eq. 1, we can estimate the background of each real sequence $\hat{\mathcal{B}}(., t)$ by substraction of these extracted domes from the sequence.

Fluorescence microscopy images are distorted by two main sources of noise; namely, photon noise (Poisson) and readout noise (Gaussian). Therefore, the stochastic model for these is be represented by a Mixed-Poisson-Gaussian (MPG) 
process [12]. Because these are generated from two different sources, these noise processes are mutually independent. To generate Poisson noise, the noise-free intensity of each pixel $\Im(\mathrm{X}, t)$ is supposed as the mean $\lambda$ of Poisson process $\mathcal{P}(\lambda)$. The readout noise can be generated by adding a gaussian noise with known mean $\mu$ and variance $\sigma^{2}$. The final noisy intensity of each pixel $\mathfrak{I}_{\eta}(\mathrm{X}, t)$ is obtained as follows.

$$
\mathfrak{I}_{\eta}(\mathrm{X}, t)=\alpha \mathfrak{I}_{\mathcal{P}}(\mathrm{X}, t)+\mathfrak{I}_{\mathcal{N}}(\mathrm{X}, t),
$$

where $\alpha>0$ is the overall gain of the imaging detector, $\mathfrak{I}_{\mathcal{P}}(\mathrm{X}, t) \sim \mathcal{P}(\mathfrak{I}(\mathrm{x}, t))$ and $\mathfrak{I}_{\mathcal{N}}(\mathrm{x}, t) \sim \mathcal{N}\left(\mu, \sigma^{2}\right)$.

Due to non-homogeneous backgrounds of the sequences and dynamic intensity of structures, the SNR of images is not constant. Instead, it can be modeled by a range between a minimum and maximum SNR. In order to fix the SNR between any desirable range, we can combine the estimated background and the generated objects using coefficients $a$ and $b$ such that $\mathfrak{I}=a \mathcal{O}+b \hat{\mathcal{B}}$. Therefore these coefficient can be calculated such that the SNR is set between a desirable range.

\subsection{Dynamic Models}

In this study we use statistical models to simulate vesicle motion dynamics. It has been shown that two linear dynamic models including random walk and nearly constant velocity motion with small accelerations can properly mimic the nonlinear motion of the vesicles in TIRFM sequences $[4,5,6]$. Random walk and constant velocity models resemble vesicle motion patterns described as tethering and docking, and linear movements, respectively [1,6]. In addition, some vesicles appear to be stationary for long periods of time. This is due to their strong attachment to the cell membrane. Hence, they are seen as immobile structures with subtle jittering motion.

As well as vesicles, there are other membrane bound subcellular structures, such as endosomes, close enough to the plasma membrane to appear in TIRFM images. Large endosomes appear as bigger structures and are usually relatively immobile. In addition, they may spawn or absorb the small structures. Thus, our sequences include these large structures.

Due to the 3-D dimensional motion of vesicles, they may exit from evanescent field. Therefore, they may either temporarily or permanently disappear from the sequences. In this simulation, we allow the structures to move outside the evanescent field and re-enter again later, thus, re-appear again in the image. Our simulation also contains new vesicles, gradually moving from outside into inside the TIRF zone.

\section{EXPERIMENTAL RESULTS}

\subsection{Parameter settings}

Generally, the framework introduced in this paper provides a simulation package which is capable of generating diverse TIRFM sequences with different quality by changing the parameters. As a sample (Fig. 2), we simulate a synthetic
TIRFM video with spatial resolution of $158 \mathrm{~nm} / \mathrm{pixel}$ and temporal resolution $9 \mathrm{fps}$. Each sequence contains on average, 80 percent small structures and 20 percent large structures. All structures are located and move inside a cell membrane (an estimated background) that is extracted from real TIRFM sequences. The small structures are generated as round or moderately elongated objects in different sizes (250-450 $\mathrm{nm})$. These structures move through the sequences using two dynamic models introduced in $\S 2.2$. Moreover, the shapes of vesicles are deformed using Eqs. 3-7 with deforming constant $\gamma=8 \mathrm{um} / \mathrm{s}$. In addition, the decay constant $\zeta=100 \mathrm{~nm}$ is used to change the intensity of moving structures. The PSF is also generated using equation 8 for an objective lens with $N A$ $=1.46$, and $\lambda=520 \mathrm{~nm}$, using the same $\mathrm{x}-\mathrm{y}$ pixel resolution. The large endosomes also appears as round or moderately elongated objects generated in different sizes (700-1600nm). These structures are stationary or have very slight jittering motion. In our simulation, these structures are able to spawn the small structures. Moreover, the small structures may be randomly fused to these large structures if they coincide in the same position. The average numbers of existing objects in each frame is 250 . In this simulation, the SNR are fixed to be between 1.5 and 8.5 .

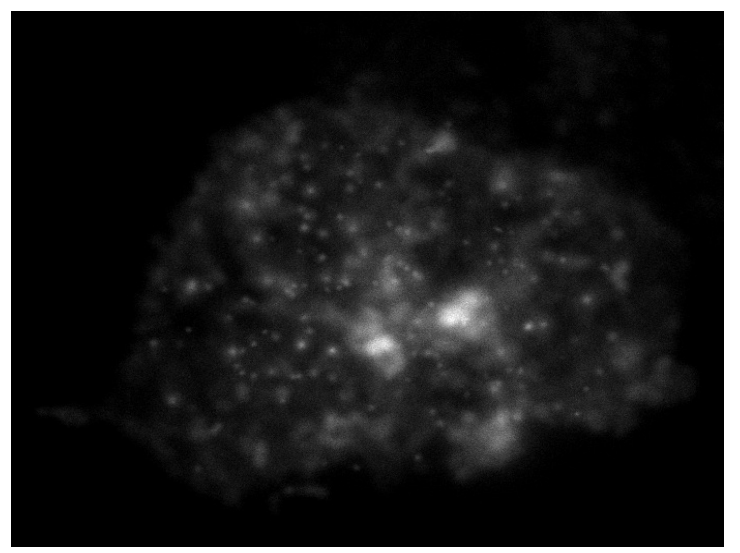

Fig. 2. A frame of the generated TIRFM sequences using the proposed framework and the aforementioned parameters.

\subsection{Evaluation}

Unfortunately, there is no straightforward way to qualitatively compare the simulated sequences with real sequences. Therefore, generated synthetic sequences were visually inspected by expert biologists and it was confirmed that our simulations are convincingly similar to real image sequences by considering both the measurement and dynamic models. Furthermore, these simulations were deemed an improvement on the current, more simplistic, synthetic models. To maximize the credibility of our simulation framework, we visually represent some of the aforementioned assumptions about the dynamic and measurement models in real TIRFM sequences and compare them with our simulation. In Fig. 3, the shape deformation of vesicles in real and synthetic sequences are shown. 

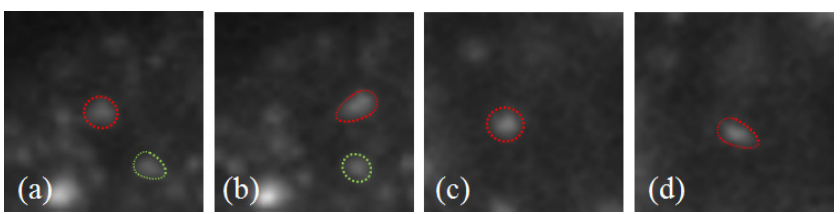

Fig. 3. Two consecutive frames from a real data ((a)-(b)) and from a synthetic data ((c)-(d)). The shapes of the vesicles deform as elongated objects with a comet tail when they move.

In order to show the accuracy of the estimator, we calculate the mean intensity of each frame for the real sequence and its estimated background. Fig. 4 shows that the estimated background accurately follows the intensity fluctuation in real TIRFM. The difference between the mean intensities is due to the removed structures (spots).

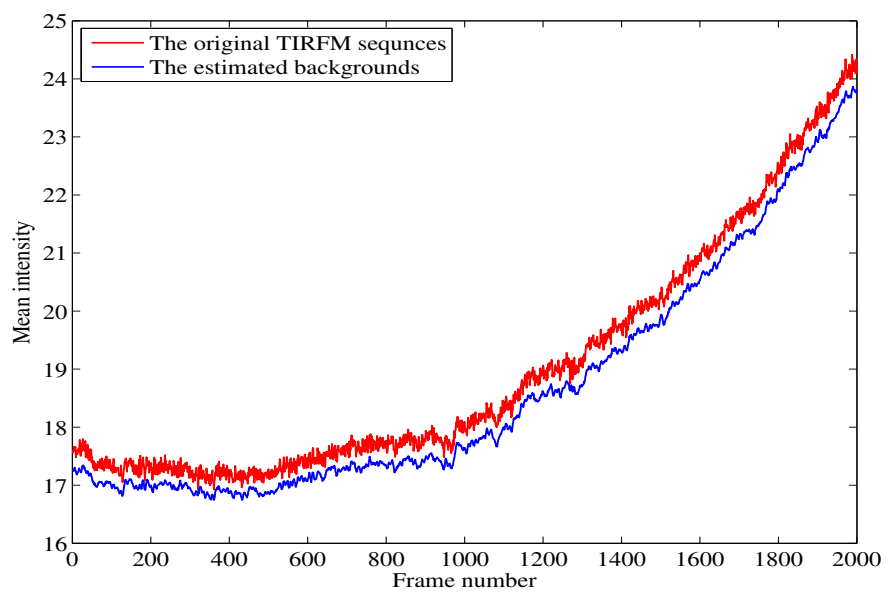

Fig. 4. The mean pixel intensity for each frame for the real data (red), and the estimated background using the MPHD method (blue).

Fig. 5(a)-(c) shows the three different dynamics commonly observed in TIRFM sequences: stationary, tethering and docking, and linear motion. Fig. 5(c) also demonstrates an example of switching dynamics of vesicles. It can be seen that the simulated dynamics using random walk and constant velocity appropriately mimic the dynamics of vesicles in real TIRFM (Fig. 5(d)-(f)).

\section{CONCLUSIONS}

Our framework greatly improve the quality of synthetically generated TIRFM sequences. However, the framework can still be improved with further simulations. Based on the imaging system and the types of green fluorescent protein, fusion of vesicles with the plasma membrane can be observed by TIRFM sequences. These fusion events are seen as sudden brightening and a rapid spreading of the intensity. In addition, other than heaped structures, there are other objects such as tubular structures in real TIRFM sequences. These structures usually have more nonlinear dynamics and complex interactions with other structures. Thus, our future work will be
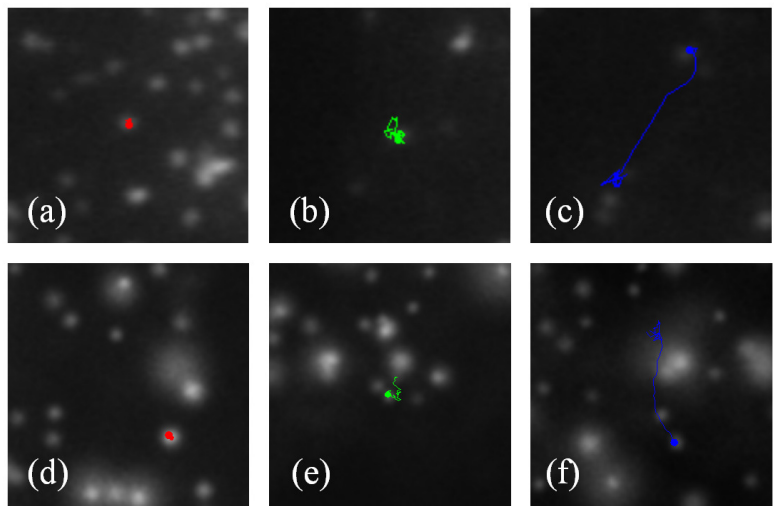

Fig. 5. (a)-(c) Manual trajectories of three different vesicles on real data and (d)-(f) its corresponding simulated trajectories on synthetic data using the proposed framework.

dissection and simulation of these processes.

\section{REFERENCES}

[1] J.G. Burchfield, J.A. Lopez, K. Mele, P. Vallotton, and W.E. Hughes, "Exocytotic vesicle behaviour assessed by total internal reflection fluorescence microscopy," Traffic, vol. 11, pp. 429-439, 2010.

[2] I. Smal, M. Loog, W. Niessen, and E. Meijering, "Quantitative comparison of spot detection methods in fluorescence microscopy," IEEE Trans. Med. Imaging, vol. 29, no. 2, pp. 282-301, 2010.

[3] I. Smal, K. Draegestein, N. Galjart, W. Niessen, and E. Meijering, "Particle filtering for multiple object tracking in dynamic fluorescence microscopy images: Application to microtubule growth analysis," IEEE Trans. Med. Imag., vol. 27, no. 6, pp. 789-804, 2008.

[4] L. Feng, Y. Xu, Y. Yang, and X. Zheng, "Multiple dense particle tracking in fluorescence microscopy images based on multidimensional assignment," J. Struct. Biol., vol. 173, no. 2, pp. 219-228, 2011.

[5] I. Smal, E. Meijering, K. Draegestein, N. Galjart, I. Grigoriev, A. Akhmanova, ME Van Royen, AB Houtsmuller, and W. Niessen, "Multiple object tracking in molecular bioimaging by raoblackwellized marginal particle filtering," Med. Image Anal., vol. 12, no. 6, pp. 764-777, 2008.

[6] S.H. Rezatofighi, S. Gould, R. Hartley, K. Mele, and W. Hughes, "Application of the IMM-JPDA filter to multiple target tracking in total internal reflection fluorescence microscopy images," Medical Imag. Comput. Computer-Assist. Interv. (MICCAI), pp. 357-364, 2012.

[7] D. Axelrod, "Cell-substrate contacts illuminated by total internal reflection fluorescence.," J. Cell biol., vol. 89, no. 1, pp. 141-145, 1981.

[8] A. Upadhyaya, J.R. Chabot, A. Andreeva, A. Samadani, and A. Van Oudenaarden, "Probing polymerization forces by using actinpropelled lipid vesicles," P. Natl. Acad. Sci. USA, vol. 100, no. 8, pp. 4521, 2003.

[9] P.A. Giardini, D.A. Fletcher, and J.A. Theriot, "Compression forces generated by actin comet tails on lipid vesicles," P. Natl. Acad. Sci. USA, vol. 100, no. 11, pp. 6493, 2003.

[10] V.F. Canales, P.J. Valle, J.E. Oti, and M.P. Cagigal, "Variable resolution with pupil masks," Opt. Commun., vol. 257, no. 2, pp. 247-254, 2006.

[11] S.H. Rezatofighi, R. Hartley, and W.E. Hughes, "A new approach for spot detection in total internal reflection fluorescence microscopy," in Proc. IEEE Int. Symp. Biomed. Imag. (ISBI), 2012, pp. 860-863.

[12] B. Zhang, J. Fadili, J.-L. Starck, and J.-C. Olivo-Marin, "Multiscale variance-stabilizing transform for mixed-poisson-gaussian processes and its applications in bioimaging," in Proc. IEEE Int. Conf. Image Process. (ICIP), 2007, pp. VI-233-VI-236. 